\title{
Les Phlébotomes (Diptera, Phlebotomidae) du Mayombe (République Populaire du Congo)
}

\author{
par G. VATTIER-BERNARD et J. TROUILLET \\ (Collaboration technique: A.-S. Bimangou) \\ Département de Biologie animale, Equipe de Bio-écologie des Vecteurs, \\ Faculté des Sciences, B.P. 69, Brazzaville, République populaire du Congo
}

\section{Résumé.}

Quinze espèces et sous-espèces de Phlébotomes sont inventoriées dans le massif forestier du Mayombe en République populaire du Congo. Neuf d'entre elles sont strictement inféodées à la forêt ombrophile équatoriale; les autres sont des espèces de forêt secondarisée, de clairière, voire de savane. Une comparaison est faite entre le peuplement phlébotomien du Mayombe et celui de plusieurs autres forêts denses congolaises.

\section{Summary.}

The Phlebotomine sandflies (Diptera) from the Mayombe area in the Popular Republic of Congo.

Fifteen species and subspecies of Phlebotomine sandflies have been inventorized in the Mayombe forest in the People's Republic of the Congo. Nine of them are confined strictly to the rain forest; the others belong to secondary forest, clearing or savannah. We have drawn a comparison between the Phlebotomine fauna in the Mayombe and those existing in several other dense Congolense forests.

A $100 \mathrm{~km}$ de l'Atlantique et parallèle à lui, la chaîne du Mayombe, large de 30 à $60 \mathrm{~km}$, représente la partie médiane d'un long massif ancien, essentiellement constitué de roches métamorphiques et couvert de forêt, s'étendant du Gabon au Cabinda.

Accepté le 26 juin 1978. 
Son relief est très accidenté : les lignes de crêtes sont étroites, les versants ravinés, les vallées profondes et encaissées (de Boissezon et coll., 1969).

Bien que d'altitude modeste (point culminant : $930 \mathrm{~m}$ environ au Mont Foungouti), ce massif ancien constitue face à l'Océan une barrière nettement plus arrosée que les régions voisines. La pluviométrie moyenne annuelle est fréquemment supérieure à $1.511 \mathrm{~mm}$ et peut atteindre $2.000 \mathrm{~mm}$.

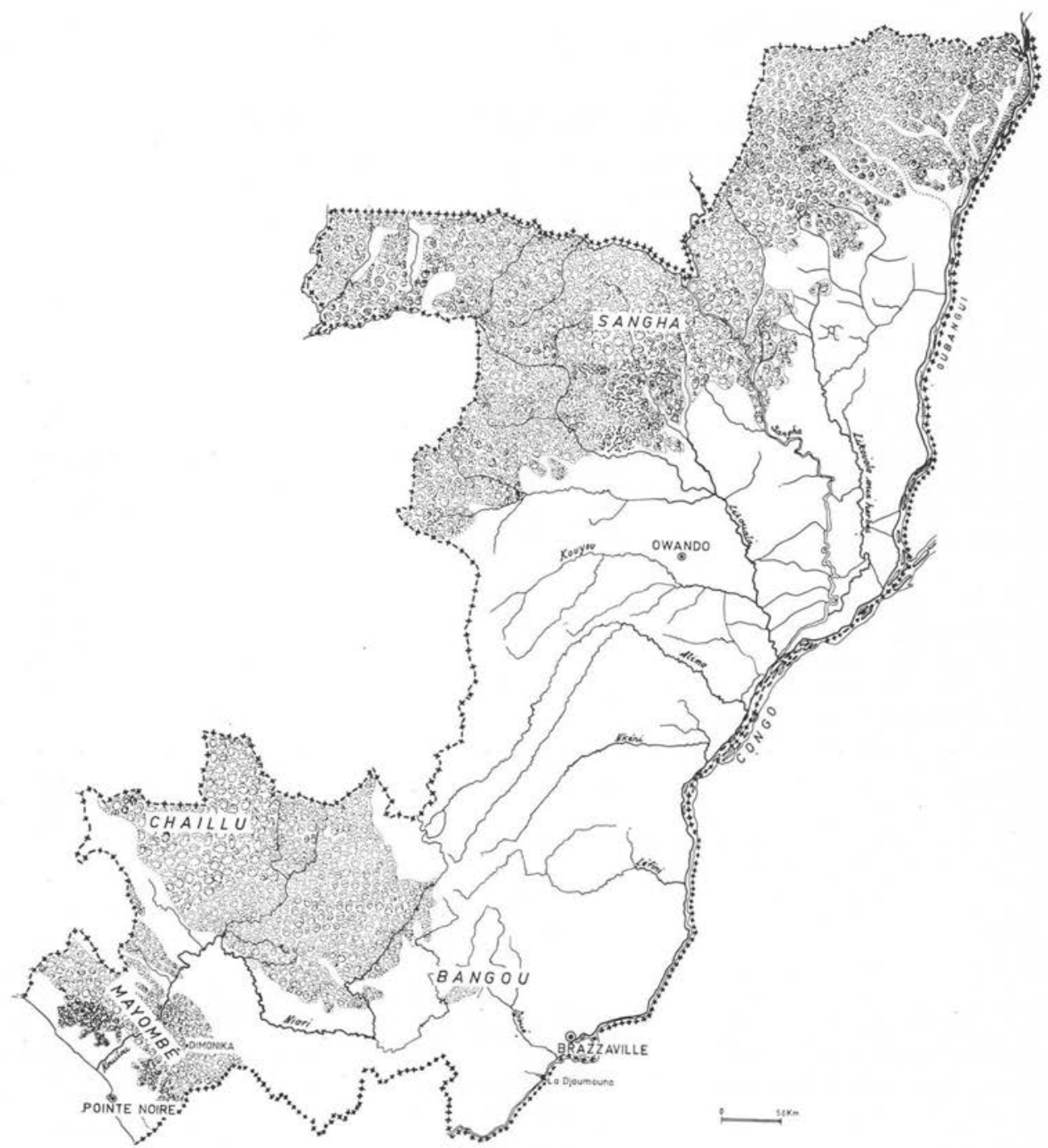

Fig. 1. Répartition de la forêt équatoriale au Congo. 


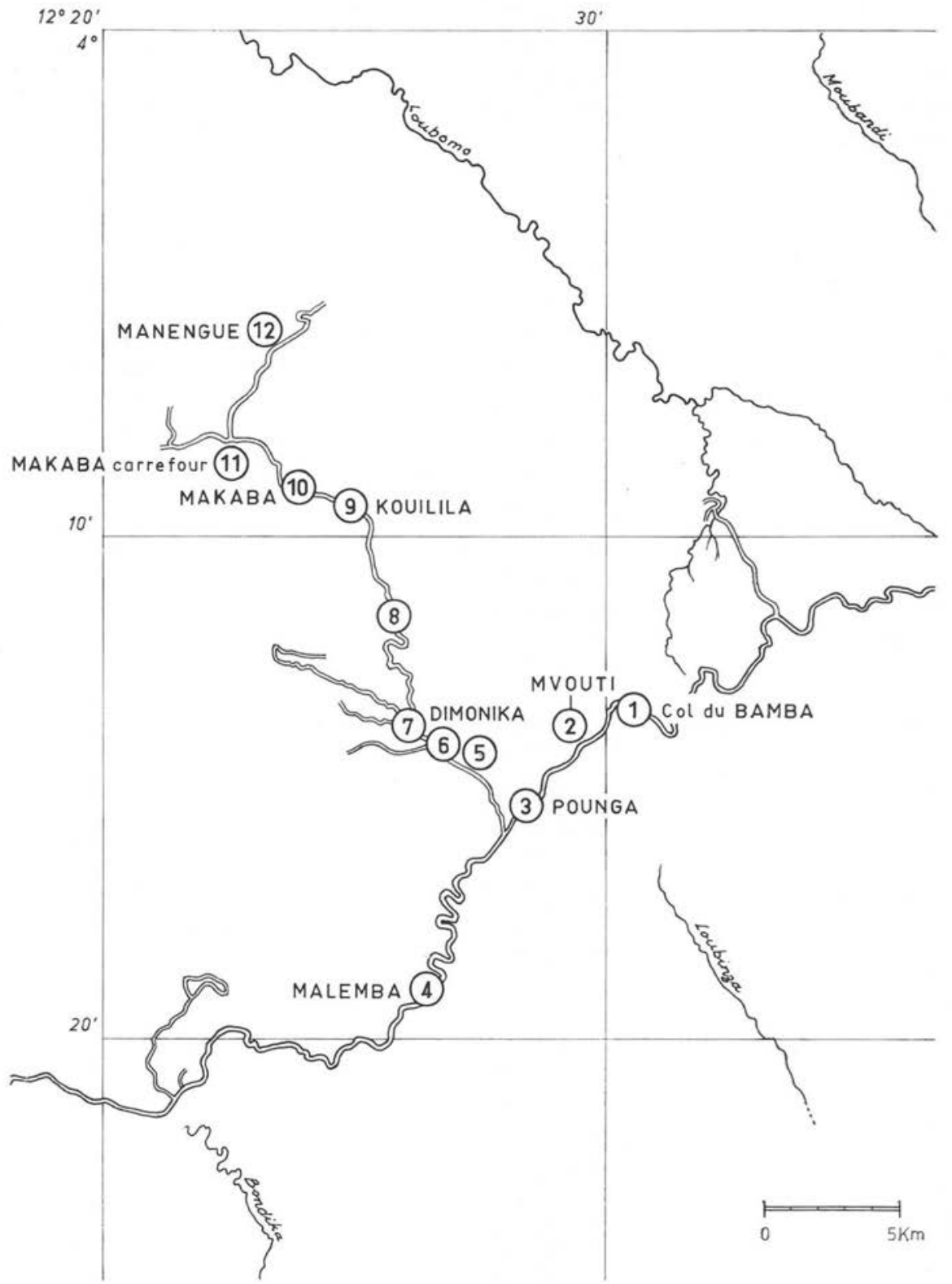

Fig. 2. Stations de capture dans la région de Dimonika. 
L'extrémité sud de la chaîne marque la limite la plus méridionale de la forêt ombrophile équatoriale gabonaise (Descoings, 1969). La forêt du Mayombe, en effet, est caractérisée par de grands arbres appartenant à des espèces variées fréquemment pourvues de contreforts. Le sous-bois clair, lorsque la forêt est demeurée primaire, devient plus difficilement pénétrable là où l'homme est intervenu pour des cultures, l'exploitation forestière et la construction des voies de communication.

\section{Stations de capture}

Entre 1972 et 1977 (1), nous avons effectué quatre missions dans la région du Mayombe (fig. 1) ; toutes ont eu lieu pendant la saison des pluies (mois de mars) ou à la fin de celle-ci (mois de mai).

La zone prospectée s'étend au nord et au sud de Dimonika (fig. 2). Situé à $5 \mathrm{~km}$ au nord de la route nationale, ce petit village est distant, à vol d'oiseau, de $9 \mathrm{~km}$ du Col Bamba, un des points les plus élevés du Mayombe $(810 \mathrm{~m})$.

Les stations de capture (tableau I), au nombre de douze, s'échelonnent de part et d'autre de deux axes sub-perpendiculaires : Col du Bamba-Malemba, Pounga-Manengué. Elles se situent dans des forêts plus ou moins en voie de secondarisation, car souvent proches de voies de communication.

Tableau I. - Stations de capture avec leurs coordonnées géographiques, les techniques de piègeage utilisées (A: capture manuelle, $B$ : piège adhésif lumineux) et le nombre de Phlébotomes récoltés.

\begin{tabular}{|c|c|c|c|c|}
\hline Stations & $\begin{array}{l}\text { Coordonnées } \\
\text { géographiques }\end{array}$ & $\begin{array}{l}\text { Type de } \\
\text { piègeage }\end{array}$ & $\begin{array}{l}\text { Nombre de } \\
\text { captures }\end{array}$ & $\begin{array}{l}\text { Nombre de } \\
\text { Phlébotomes } \\
\text { récoltés }\end{array}$ \\
\hline $1 \mathrm{Col}$ du Bamba & $4^{\circ} 14^{\prime} \mathrm{S}-12^{\circ} 31^{\prime} \mathrm{E}$ & A & 1 & 16 \\
\hline 2 M'Vouti ................... & $4^{\circ} 15^{\prime} \mathrm{S}-12^{\circ} 30^{\prime} \mathrm{E}$ & A & 2 & 79 \\
\hline $\begin{array}{l}3 \text { Pounga ...................... } \\
4 \text { Entre Malemba et Poun- }\end{array}$ & $4^{\circ} 16^{\prime} \mathrm{S}-12^{\circ} 28^{\prime} \mathrm{E}$ & A & 1 & 76 \\
\hline 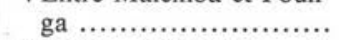 & $4^{\circ} 19^{\prime} \mathrm{S}-12^{\circ} 27^{\prime} \mathrm{E}$ & A & 1 & 99 \\
\hline 5 Entre Dimonika et & & & & \\
\hline Pounga...$\ldots \ldots \ldots \ldots \ldots$ & $4^{\circ} 15^{\prime} \mathrm{S}-12^{\circ} 27^{\prime} \mathrm{E}$ & A & 3 & 281 \\
\hline 6 Dimonika source ......... & $4^{\circ} 14^{\prime} \mathrm{S}-12^{\circ} 27^{\prime} \mathrm{E}$ & A & 3 & 153 \\
\hline 7 Dimonika ................. & $4^{\circ} 14^{\prime} \mathrm{S}-12^{\circ} 26^{\prime} \mathrm{E}$ & $A+B$ & 3 & $85+58$ \\
\hline 8 Entre Kouilila et Dimo- & & & & \\
\hline 9 Entre Makaba et Kouilila & $\begin{array}{l}4^{\circ} 12^{\prime} \mathrm{S}-12^{\circ} 26^{\prime} \mathrm{E} \\
4^{\circ} 10^{\prime} \mathrm{S}-12^{\circ} 25^{\prime} \mathrm{E}\end{array}$ & $\begin{array}{l}\mathrm{A} \\
\mathrm{A}\end{array}$ & $\begin{array}{l}1 \\
1\end{array}$ & $\begin{array}{r}136 \\
16\end{array}$ \\
\hline 10 Makaba .................... & $4^{\circ} 09^{\prime} \mathrm{S}-12^{\circ} 24^{\prime} \mathrm{E}$ & $\mathrm{A}$ & 2 & 90 \\
\hline 11 Makaba carrefour ........ & $4^{\circ} 08^{\prime} \mathrm{S}-12^{\circ} 23^{\prime} \mathrm{E}$ & $A+B$ & 2 & $32+1$ \\
\hline 12 Manengué ............... & $4^{\circ} 06^{\prime} \mathrm{S}-12^{\circ} 23^{\prime} \mathrm{E}$ & $A+B$ & 4 & $466+83$ \\
\hline
\end{tabular}

(1) Travail effectué dans le cadre de l'Equipe Pluridisciplinaire de Biologie forestière tropicale de Dimonika, depuis 1977. 


\section{Techniques de capture}

Deux procédés de capture ont été utilisés :

- la capture manuelle, il s'agit, d'une main, d'éclairer le support (mur, arbre, anfractuosité...) avec une lampe de poche et, de l'autre, de capturer l'insecte en le coiffant d'un tube en verre de petite taille $(65 \mathrm{~mm}$ de long). Le tube est ensuite fermé par un bouchon en liège. Ce mode de capture a été utilisé en forêt ;

- le piège adhésif lumineux (Rioux et Golvan, 1969) constituć d'une planche de contreplaqué portant 6 réflecteurs de lampe de poche alimentés par une batterie de 6 volts. Devant chaque lampe, une feuille de papier $(20 \times 20 \mathrm{~cm})$ imbibée d'huile de ricin, est tendue.

Ce deuxième procédé, beaucoup moins précis que la capture manuelle, ne donne aucun renseignement sur les gîtes de repos. De plus, en vue d'une interprétation satisfaisante des résultats, il est important de noter que, pour des raisons indépendantes de notre volonté, nous avons été contraints de placer le piège en lisière de forêt. Autrement dit, les espèces de clairière, voire de savane, ont été attirées en même temps que les espèces de la forêt voisine.

\section{Résultats}

Aucune étude n'avait été faite jusqu'alors sur la faune phlébotomienne du Mayombe. Ces premières prospections ont permis :

- de récolter 1671 phlébotomes et d'inventorier 15 espèces et sous-espèces, soit $41 \%$ des espèces constituant la faune phlébotomienne actuellement recensée au Congo. Ces espèces sont les suivantes:

Grassomyia ghesquierei Parrot, 1929.

Sergentomyia (Spelaeomyia) emilii Vattier, 1966.

Sergentomyia (Sergentomyia) bedfordi medius Kirk et Lewis, 1950.

Sergentomyia (Sergentomyia) bedfordi congolensis Bequaert et Walravens, 1930.

Sergentomyia (Sergentomyia) impudica Abonnenc, 1968.

Sergentomyia (Sergentomyia) schwetzi Adler, Theodor et Parrot, 1929.

Sergentomyia (Sergentomyia) crosarai Parrot et Wanson, 1946.

Sergentomyia (Rondanomyia) ingrami Newstead, 1912.

Sergentomyia (Rondanomyia) collarti Adler, Theodor et Parrot, 1929.

Sergentomyia (Rondanomyia) dureni Parrot, 1934.

Sergentomyia (Rondanomyia) dyemkoumai Abonnenc, 1964.

Sergentomyia hamoni Abonnenc, 1958.

Sergentomyia simillima Newstead, 1914.

Sergentomyia moreli Abonnenc et Hamon, 1958.

Sergentomyia grjebinei Vattier-Bernard, 1971. 
- de mettre en évidence, une fois de plus, la nécessité d'utiliser plusieurs modes de piégeage (tableau II). Certaines espèces, notamment $G$. ghesquierei et $S$. dureni ne sont pratiquement jamais capturées à la main quel que soit le biotope prospecté (VattierBernard et Trouillet, sous-presse).

Tableau II. - Comparaison des résultats en fonction du procédé de piégeage.

\begin{tabular}{|c|c|c|c|c|}
\hline \multirow{2}{*}{$\begin{array}{c}\text { Espèces } \\
\text { S. hamoni } \ldots \ldots \ldots \ldots \ldots \ldots\end{array}$} & \multicolumn{2}{|c|}{$\begin{array}{l}\text { Nombre de Phlébotomes } \\
\text { capturés à la main }\end{array}$} & \multicolumn{2}{|c|}{$\begin{array}{l}\text { Nombre de Phlébotomes } \\
\text { capturés au piège } \\
\text { adhésif lumineux }\end{array}$} \\
\hline & 800 & $52,32 \%$ & 0 & - \\
\hline S. bedfordi $\ldots \ldots \ldots \ldots \ldots$ & 423 & $27,66 \%$ & 5 & $3,52 \%$ \\
\hline S. moreli ..................... & 263 & $17,20 \%$ & 1 & $0,70 \%$ \\
\hline S. ingrami ................. & 15 & $0,98 \%$ & 1 & $0,70 \%$ \\
\hline S. impudica ............... & 9 & $0,59 \%$ & 1 & $0,70 \%$ \\
\hline S. collarti .................. & 8 & $0,52 \%$ & 15 & $10,56 \%$ \\
\hline S. dyemkoumai ........... & 7 & $0,46 \%$ & 0 & - \\
\hline S. grjebinei ............... & 2 & $0,13 \%$ & 0 & - \\
\hline S. crosarai $\ldots \ldots \ldots \ldots \ldots$ & 1 & $0,06 \%$ & 0 & - \\
\hline S. schwetzi ............... & 1 & $0,06 \%$ & 0 & - \\
\hline S. emilii .................... & 0 & - & 1 & $0,70 \%$ \\
\hline S. simillima $\ldots \ldots \ldots \ldots \ldots$ & 0 & - & 2 & $1,41 \%$ \\
\hline S. dureni .................... & 0 & - & 1 & $0,70 \%$ \\
\hline G. ghesquierei ............. & 0 & - & 115 & $80,98 \%$ \\
\hline Total ......................... & 1529 & & 142 & \\
\hline
\end{tabular}

- de comparer la faune phlébotomienne du Mayombe à celle de plusieurs autres forêts ombrophiles congolaises (tableau III) où de nombreuses captures manuelles ont été effectuées.

Tableau III. - Peuplement phlébotomien des principales forêts ombrophiles congolaises.

\begin{tabular}{|c|c|c|c|c|c|}
\hline Espèces & Mayombe & $\begin{array}{l}\text { Haute } \\
\text { Sangha }\end{array}$ & Bangou & $\begin{array}{c}\text { Le } \\
\text { Chaillu }\end{array}$ & $\begin{array}{c}\text { La Djou- } \\
\text { mouna }\end{array}$ \\
\hline S. moreli ........................... & $\begin{array}{l}2^{*}, 3,4,5,6,7,8,10 \\
11,12\end{array}$ & + & + & + & + \\
\hline S. hamoni ........................ & $2,3,4,5,6,7,10,11,12$ & + & + & + & + \\
\hline S. bedfordi medius $\ldots \ldots \ldots \ldots \ldots$ & $2,4,6,7,8,9,10,11,12$ & + & + & + & + \\
\hline S. bedfordi congolensis............. & $1,2,5,6,7,10,12$ & + & + & + & + \\
\hline S. ingrami $\ldots \ldots \ldots \ldots \ldots \ldots \ldots \ldots \ldots \ldots \ldots \ldots \ldots \ldots \ldots$ & $4,5,6,7,10,12$ & + & + & + & + \\
\hline 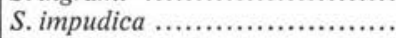 & $4,6,12$ & + & + & + & + \\
\hline S. dyemkoumai ................... & 2 & + & + & + & \\
\hline S.grjebinei ...................... & 3,12 & + & + & & \\
\hline 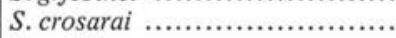 & 8 & & + & & + \\
\hline S. emilii ........................... & 12 & + & & + & + \\
\hline S. simillima ....................... & 7 & & & & \\
\hline S. dureni .......................... & 7 & + & & & + \\
\hline 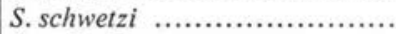 & 5 & & + & & + \\
\hline 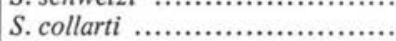 & $7,9,11,12$ & & & & + \\
\hline G. ghesquierei ..................... & $7,11,12$ & & & & + \\
\hline
\end{tabular}




\section{Commentaires}

A la lecture des tableaux II et III, il apparaît nettement que les espèces les plus fréquentes dans le Mayombe sont également inféodées aux forêts ombrophiles de la Haute Sangha (Vattier-Bernard et Bimangou, 1974), du Chaillu et de Bangou (VattierBernard et Trouillet, sous-presse). Elles sont encore présentes à $21 \mathrm{~km}$ de Brazzaville (Carte 1), à la Djoumouna, ancienne forêt primaire, réduite à une forêt galerie secondarisée du fait du développement des cultures et du déboisement que celles-ci entraînent.

Ces espèces forestières sont essentiellement: $S$. moreli, S. hamoni, S. bedfordi, S. ingrami, S. impudica, S. dyemkoumai, S. grjebinei, S. crosarai, S. emilii.

$S$. hamoni et $S$. bedfordi prédominent nettement alors que $S$. grjebinei, $S$. crosarai et $S$. emilii n'ont été récoltés qu'en un ou deux exempiaires. A l'exception de $S$. emilii, qui a d'abord été découvert dans des grottes (Vattier-Bernard, 1970) ces trois espèces ne sont connues qu'en milieu forestier dense où elles ne constituent toujours qu'un très faible pourcentage du peuplement phlébotomien $(0,05$ à $0,16 \%)$. Il s'agirait donc d'espèces à très faible densité de population, voire d'espèces relictuelles s'attardant dans quelques microbiotopes.

La présence de $S$. schwetzi, espèce abondante en savane, dans les plantations et les habitants est, semble-t-il, accidentelle en forêt ombrophile ; elle peut être due à un effet de lisière (Dajoz, 1970).

$S$. collarti et $S$. dureni ne peuvent être étroitement inféodés à la forêt dense. Au Congo (Vattier-Bernard et Trouillet, sous-presse), ces espèces ont été récoltées en savane, dans les plantations et les abris sous-roches, en forêt galerie et dans des îlots forestiers très secondarisés. $S$. dureni, attiré par la lumière, s'aventure dans les habitations. Quelques spécimens ont été capturés en plein cœur de Brazzaville, autour de lampes de chevet.

G. ghesquierei nous est connu au Congo, essentiellement grâce à sa phototaxie positive. Dans le Mayombe, il a été capturé en lisière de forêt; dans d'autres régions, nous l'avons pris, toujours au piège lumineux, dans des villages ou aux abords de ceux-ci. Une larve a été trouvée au pied d'un Oranger (Trouillet, 1976) dans un sol riche en matière organique, sous un épais tapis végétal. Ce gîte se situait près d'un marigot, au sein même d'un village. Abonnenc (1972) n'estime-t-il pas du reste que les espèces de Grassomyia vivent le plus souvent dans la végétation herbacée, non loin des points d'eau.

\section{Bibliographie}

Abonnenc E. (1972): Les Phlébotomes de la région éthiopienne (Diptera, Psychodidae). Mem. O.R.S.T.O.M., $\mathrm{n}^{\circ}$ 55, 289 p.

Boissezon P. de, Martin G., Gras F. (1969): Les sols du Congo, in Atlas du Congo. O.R.S.T.O.M., Brazzaville. 
Descoings B. (1969): Esquisse phytogéographique du Congo, in Atlas du Congo. O.R.S.T.O.M., Brazzaville.

Rioux J.A., Golvan Y.J. (1969) : Epidémiologie des Leishmanioses dans le Sud de la France. Monographie de l'I.N.S.E.R.M., 37, Paris, 221.

Trouillet J., (1976) : Sergentomyia (Grassomyia) ghesquierei Parrot (Diptera, Phlebotomidae). Etude morphologique des stades préimaginaux et notes bioécologiques. Cah. O.R.S.T.O.M., Ser. Entomol. Med. Parasitol., 14, 347-356.

Vattier-Bernard G. (1970): Contribution à l'étude systématique et biologique des Phlébotomes cavernicoles en Afrique intertropicale. Cah. O.R.S.T.O.M., Ser. Entomol. Med. Parasitol., 8, 175-288.

Vattier-Bernard G., Bimangou A.S. (1974) : Contribution à la faune de la République Populaire du Congo. IX. - Phlébotomes de la Sangha et de la Cuvette (Diptères, Psychodidae). Ann. Univ. Brazzaville, 10 (C), 91-109.

Vattier-Bernard G., Trouillet J. (1976) (sous-presse) : Contribution à la faune de la République Populaire du Congo. XII. - Phlébotomes (Diptera, Phlebotomidae) de la Région du Pool. Inventaire, Répartition géographique et Etude écologique. Ann. Univ. Brazzaville, 12 (C), ... - ..

Vattier-Bernard G., Trouillet J. (1976) (sous-presse) : Contribution à la faune de la République Populaire du Congo. XIII. Phlébotomes (Diptera, Phlebotomidae) de la Bouenza, de la Lekoumou et du Niari. Inventaire, Répartition géographique et notes écologiques. Ann. Univ. Brazzaville, $12(C), \ldots-\ldots$ 